\title{
Treatment of a lung lobectomy patient with severe post-surgical infection in the anterior thoracic wall by multiple debridement and drainage procedures: a case report
}

\author{
Jie Dai $^{1}$, Patrick Greiffenstein ${ }^{2}$, Francesco Petrella ${ }^{3,4}$, Jae Jun Kim ${ }^{5}$, Giuseppe Marulli ${ }^{6}$, Yong Fang ${ }^{7}$, \\ Yiming Zhou ${ }^{1}$
}

${ }^{1}$ Department of Thoracic Surgery, Shanghai Pulmonary Hospital, Tongji University School of Medicine, Shanghai, China; ${ }^{2}$ Department of Surgery, Louisiana State University Health Science Center, New Orleans, LA, USA; ${ }^{3}$ Department of Thoracic Surgery, IRCCS European Institute of Oncology, Milan, Italy; ${ }^{4}$ Department of Oncology and Hemato-Oncology, University of Milan, Milan, Italy; ${ }^{5}$ Department of Thoracic and Cardiovascular Surgery, Uijeongbu St. Mary's Hospital, College of Medicine, The Catholic University of Korea, Seoul, Korea; ${ }^{6}$ Thoracic Surgery Unit, Department of Emergency and Organ Transplantation, University Hospital of Bari, Bari, Italy; ${ }^{7}$ Department of Tuberculosis, Shanghai Pulmonary Hospital, Tongji University School of Medicine, Shanghai, China

Correspondence to: Yiming Zhou. Department of Thoracic Surgery, Shanghai Pulmonary Hospital, Tongji University School of Medicine, 507 Zhengmin Road, Shanghai 200433, China. Email: zymany@163.com.

Submitted Sep 22, 2020. Accepted for publication Oct 27, 2020.

doi: 10.21037/jtd-20-2946

View this article at: http://dx.doi.org/10.21037/jtd-20-2946

\section{Introduction}

Wound infection is a common post-surgical complication following lung lobectomy (1), which, in most cases, is localized to the area around the incision. In this manuscript, we report a case in which a mid-aged male patient suffered from a postoperative infection after lobectomy for noncancer lung disease. At the initial phase, the patient developed pleural empyema and wound infection 3 months after operation, and was treated by closed thoracic drainage. Unfortunately, the contamination was still out of control, and spread to anterior thoracic wall, sternum, and both sides of the costal arch. Multiple procedures of debridement were subsequently performed, with dressings changed on a daily basis. The patient healed completely after over three months of treatment, and experienced a good quality of life at one-year follow up. We present the following article in accordance with the CARE reporting checklist (available at http://dx. doi. org/10. 21037/jtd-20-2946).

\section{Case presentation}

The patient was a 44-year-old male who underwent a lobectomy of the left lower lobe in a local hospital in February 2009. After the surgery, the lesion was pathologically identified as bronchiectasis with a concomitant Aspergillus infection in the left lower lung. The patient made an uncomplicated recovery from a subsequent thoracotomy to stop the bleeding on the day after the lobectomy. However, 3 months after the surgery, the patient suffered from a pleural empyema along with a wound infection and was treated through closed thoracic drainage; the pathogen was not identified. By June 2009, although the condition of the pleural empyema had greatly improved, the wound infection had not shown significant improvement. At this time, the patient underwent subcutaneous drainage instead of closed thoracic drainage. However, after the drainage procedure, the patient exhibited increased inflammation in the anterior thoracic cavity.

In September 2009, the patient was admitted to our hospital and described intense chest pain; he scored 3 on the Performance Status (PS) scale. A chest computed tomography (CT) scan revealed partial damage in a portion of the bilateral ribs adjacent to the sternum, necrosis in the 7-9 costal arch and lower sternum, and an infection in

\footnotetext{
$\wedge$ ORCID: 0000-0003-4316-4243.
} 


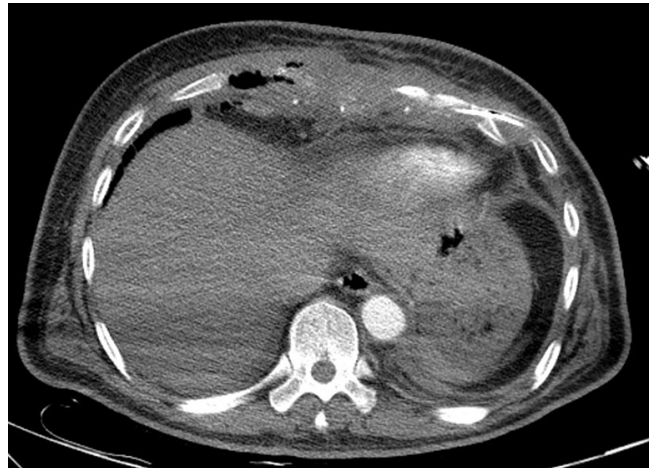

Figure 1 Pre-surgical CT scan of the anterior chest indicating an infection of the anterior thoracic wall along with a fractured sternum and ribs.



Figure 2 Pre-surgical 3D-CT reconstruction of the anterior chest revealing damage to the sternum and the bilateral ribs.

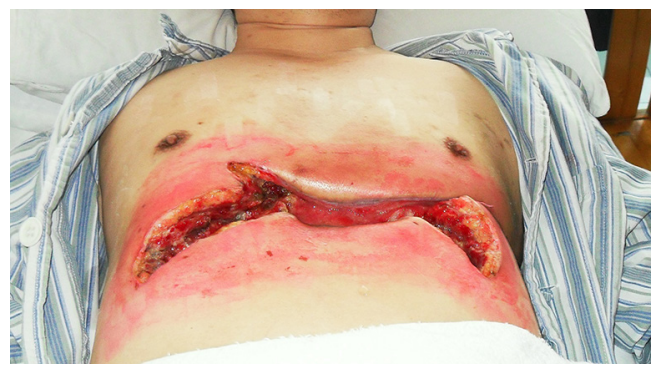

Figure 3 Intensive damage to the thoracic wall after three debridements.

the anterior thoracic wall (Figures 1,2). After admission, debridement was performed on September 16, 2009, via open operation. During the debridement process, a significant amount of necrotic tissue and pus were removed from an area below the skin and extending to the interior wall of the chest cavity; the ribs, rib cartilage, and lower sternum were found to be fractured. The patient's xiphoid, lower sternum, anterior portions of ribs 6-9, and the 79 costal arch were all excised and the infected tissue between the ribs was cleaned to expose a part of the pleura. Due to the infection flanking the original wound, drainage was not free-flowing as of October 19, 2009; therefore, on November 19, 2009, further debridement was performed towards the lower left and lower right costal arches. The infected tissue generated during the three surgeries was sent for pathological examination, which revealed inflammation in the rib cartilage and connective tissue. However, the investigation failed to identify Aspergillus or evidence of any other specific infection both during and after the surgery despite multiple attempts of identifying the pathogen by culturing pus with common media as well as media for fungi, Mycobacterium tuberculosis, and anaerobes. After the surgeries, the patient exhibited considerable damage to the thoracic wall (Figure 3). Gauze soaked with normal saline was used to cover the wounds post-operatively, and dressings were applied and changed twice daily.

After 104 days of treatment, the wound healed completely, and the patient was discharged from the hospital in January 2010. Follow-up visits were performed during one year after the surgeries and revealed a PS score of 0 , no recurring infection, and excellent daily conditions (Figure 4). The patient expressed satisfaction with his quality of life and showed no apparent functional damage except for an occasional complaint due to discomfort in the bilateral hypochondrium. The original wound area was clean and showed no signs of infection.

All procedures performed in studies involving human participants were in accordance with the ethical standards of the institutional and/or national research committee(s) and with the Helsinki Declaration (as revised in 2013). Written informed consent was obtained from the patient for publication of this manuscript and any accompanying images.

\section{iMDT discussion}

\section{Discussion among surgeons and physicians from Shanghai Pulmonary Hospital Tongii University}

For standard thoracic surgery, postoperative infections include chest wound infection, ipsilateral pneumonia, 

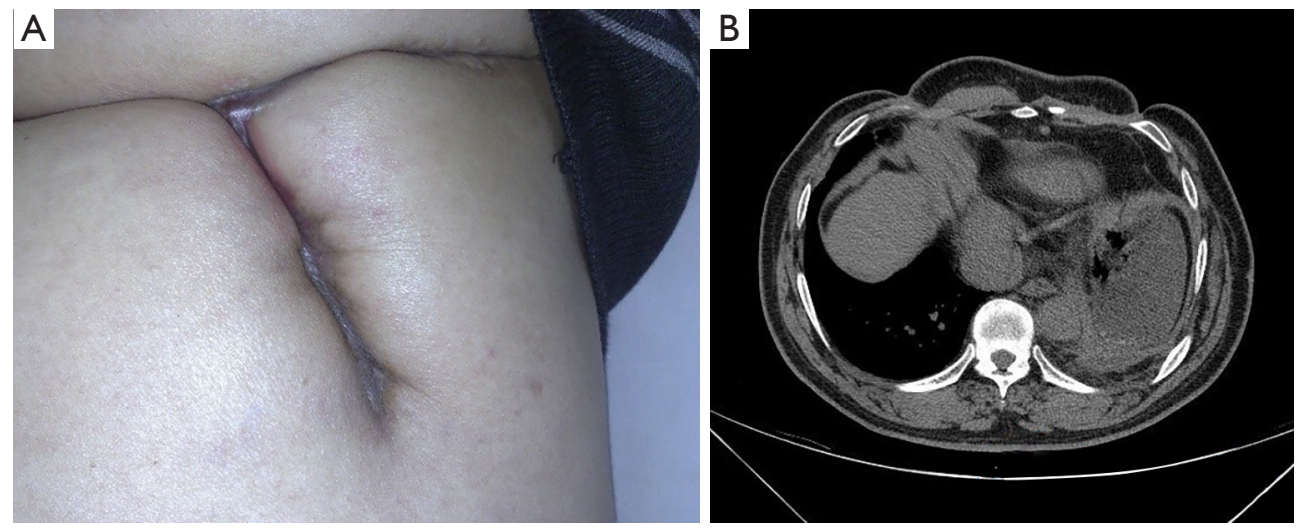

Figure 4 A follow-up visit 1 year after the surgery proved excellent wound healing and CT scan shows no sign of infection.

and postoperative empyema, which constituted $14-16 \%$ after lung resection (2). Several factors, both patientrelated and treatment-related, have been identified to increase the risk of infectious complications, including male gender, coexisting diabetes mellitus, inadequate antibiotic prophylaxis, and major anatomic resection (2). However, large-scale infection of the thoracic wall of this type resulting from pleural empyema is extremely rare among post-lobectomy patients. It may present as localized chest pain, erythema, and swelling, while signs of systemic toxicity include fever, sweating, and hypotension (3). It is reported that most of deep chest wall infections were associated with chest tube placement for empyema, or spontaneous drainage of the empyema necessitans (4). Treatment depends on the magnitude and location of infection, mainly include antibiotic administration, surgical debridement, and dressing changes.

\section{Department of Thoracic Surgery}

It has been acknowledged that drainage is a standard treatment for superficial surgical site infection (as the patient was presented initially at the local hospital), and the wound should be scrutinized if any evidence of infection from deeper tissue is indicated (3). With the progress of chest wall infection and due to inadequate drainage of empyema, sternum can be involved. Typically, complete debridement and subsequent myocutaneous flap transplantation or filling with artificial materials could cure sternum infection $(5,6)$. In this case, the patient suffered from an intense infection of an unidentified pathogen encompassing not only the sternum but the ribs. Debridement of the necrotic tissue is the only way to survive the patient. Theoretically, antibiotics may not reach the infected region due to tissue ischemia caused by a localized hypercoagulable state, but can remove potential infection seeding sources. The extent of debridement requires resection of all infected and devascularized tissue, and sometimes should continue for several times, to prevent further recurrence. Therefore, debridement can be massive, and future soft tissue coverage ought to be planned in advance. The original treatment plan for this patient was to control the infection through open dressings and to repair the lesion via myocutaneous flap transplantation.

\section{Department of Internal Medicine}

This case is challenging not only because of the extensive infection in the anterior chest wall involving sternum and bilateral costal arch, but for the unknown pathogen and consequent ineffective antibiotic/antifungal treatment. Theoretically, fungal infection should be initially suspected due to the microbiological evidence in the pathological specimen. Additionally, the underlying causes for bronchiectasis/aspergillus infection should be further explored. The patient's comorbid condition, such as an immune-related disease and use of corticosteroids, ought to be evaluated to rule out that the patient was in an immunocompromised status. Although the examination of pathogen covered common bacteria as well as fungi, mycobacterium tuberculosis, and anaerobes, the possibility of parasite infection should be examined considering the patient's occupational exposure in the abattoir. If these approaches failed to provide any definitive diagnosis, next gene sequencing may be the only viable option for identifying the causative organism(s). 
Several issues regarding the diagnosis and treatment of this patient were further discussed as follows

Question 1: In the whole treatment process, all microbiological tests cannot determine the specific pathogen, whether there is a method to identify the pathogen

\section{Expert opinion 1: Dr. Francesco Petrella}

Sometimes, even in patients with purulent secretions and empyema, microbiological tests fail to show the pathogen. Anyway, if there is a clear infected surgical field, wide spectrum antibiotic therapy is recommended; considering the previous recent infection by Aspergillus, antifungal therapy is also recommended.

\section{Expert opinion 2: Dr. Giuseppe Marulli}

The inability to find a specific pathogen is not uncommon in case of wound/chest wall infection, especially after multiple antibiotic treatments. Sometimes, if fever and leukocytosis occur blood culture at the peak of fever may be positive, otherwise large spectrum antibiotics have to be used.

\section{Expert opinion 3: Dr. Fae fun Kim}

Topic on this case is very important. However, there are few studies in the field of thoracic surgery and many studies have been performed at the fields of orthopedics, especially insertion of implant surgery. Therefore, we can apply their finding to our thoracic fields. We call this condition as culture-negative infection. The absence of culturable bacterial pathogens is a common dilemma for surgeons. Potential causes of culture-negative infection include prior antimicrobial therapy; the presence of fastidious or slowgrowing microorganisms such as fungi, mycobacteria, Mycoplasma spp., and Legionella spp.; infection caused by mundane bacteria that may be dismissed as "contaminants"; factitious infection; and others. We can diagnose it by the laboratory findings such as C-reactive protein (CRP) and erythrocyte sedimentation rate (ESR), leucocyte esterase, alpha-defensin assay, D-dimer, interleukin-6 (IL-6) assay, and fluid D-lactate. Beside an extended culture, we can identify the pathogen with the advent of polymerase chain reaction, mass spectrometry and next generation sequencing. Identifying the causative organism(s) may become easier but such techniques are not readily available and are very costly.

\section{Expert opinion 4: Dr. Patrick Greiffenstein}

The fulminant nature of NSTI mandates immediate treatment by broad-spectrum IV antibiotics as well as early and aggressive local debridement of affected tissue. The conundrum is that by initiating systemic antimicrobial therapy prior to obtaining a tissue sample for culture, providers are affecting the ability to grow the causative organisms in culture. Whenever possible cultures should be taken from the tissue, as well as from blood, prior to the initiation of antimicrobial therapy. This can be exceedingly difficult to coordinate especially when the infected tissue is closed within the skin (subcutaneous infection without an open wound). Another consideration, is for hospital microbiology laboratories to continue to pursue more advanced methods for organism identification that precludes cultures. Modern methods such as biosensors, quantitative polymerase chain reaction (qPCR), genetic sequencing, and rRNA clone libraries can directly identify microbial particles more effectively and do not rely on growing viable organisms (7). The investment in such technologies would not be specific for the treatment of NSTI, but would rather aid hospitals in responding to the need of providers to more accurately tailor therapy and should be considered a justifiable expense. With the information given, it is unclear what the methodology of the microbiology lab was in this particular case, but the timeline (at least 15 years ago from the date of this submission) many of the modern technology mentioned above was not available.

\section{Question 2: Is there a better way to deal with such a} large-scale infection?

\section{Expert opinion 1: Dr. Francesco Petrella}

Although surgical debridement, drainages and wide spectrum antimicrobial therapy are the corner stones of treatment, vacuum therapy (VAC therapy) in the later stage of treatment could be taken into consideration to improve the healing process.

\section{Expert opinion 2: Dr. Giuseppe Marulli}

When the bone is involved by infection a conservative treatment by drugs and drainage is usually invariably ineffective. Osteomyelitis often requires large chest wall resections in order to limit the spread of infection through the bone marrow way. In this case, another important point is the reconstruction: the use of synthetic materials is contraindicated due to the high risk of contamination, for that reason if a rigid material is necessary to avoid a flail chest a bone auto/allograft or titanium plates may have less risk to fail. Additionally, a muscular/musculocutaneous or omental vital flaps could increase the chance of healing. 


\section{Expert opinion 3: Dr. Fae fun Kim}

There is no royal road to managing this condition. I think that the general rule is the most important. A timely diagnosis, aggressive and meticulous debridement, high vacuum closed-suction drain, routine and adequate use of antibacterial agents are the keys to successfully resolving infection. If we follow this general rule, we can effectively manage this condition. In addition, there are studies reporting that "Culture-negative infections without osteomyelitis are less severe than culture-positive infections".

\section{Expert opinion 4: Dr. Patrick Greiffenstein}

NSTI can be insidious in its progression, despite the assumption that all behave as the classic "necrotizing fasciitis" presentation of rapidly progressive spread of necrosis. Therefore, providers should be highly suspicious of wounds that are unresponsive to antibiotic therapy and limited drainage or debridement. Such infections can have the appearance of cellulitis or simple abscesses while residual necrotic tissue remains under the skin or fascial surface serving as a nidus for progression of the disease. Therefore, wounds that do not respond to a combination of systemic antibiotics and source control (with drainage or limited debridement) within 24-48 hours should be explored or re-explored surgically. Unfortunately, there is no substitute to wide local debridement of all grossly affected tissue.

\section{Question 3: We learned that the patient died of} ruptured aortic aneurysm 6 years after surgery, whether there was a potential association between the aneurysm and the patient's infection and debridement?

\section{Expert opinion 1: Dr. Francesco Petrella}

Considering that aortic arch aneurysm rupture occurred 6 years after surgery, I do not think there is any relation between the past infection and the aortic rupture; on the contrary, if the aortic rupture had happened soon after the infection, I would have considered a closer causal relation between the two clinical events.

\section{Expert opinion 2: Dr. Giuseppe Marulli}

The angio-tropism of Aspergillus is well known so that a possible damage of the aorta could be happened during the complications of surgery, however a long time passed between the two events and a clear etiologic relationship is hard to find.

\section{Expert opinion 3: Dr. Fae fun Kim}

Factors that can contribute to an aneurysm's development include atherosclerosis, Genetic conditions (Marfan syndrome, vascular Ehlers-Danlos, Loeys-Dietz and Turner syndromes), Inflammatory conditions (giant cell arteritis and Takayasu arteritis), aortic valve disease, untreated infection, such as syphilis or salmonella, and traumatic injury. Infection can develop in the aorta two different ways: Mycotic aneurysm and Aortic graft infection. A septic embolism that results in a mycotic aneurysm is often the result of inflammation in infective endocarditis that is generally caused by bacteria. Infection in this patient is possibly associated with aortic aneurysm.

\section{Expert opinion 4: Dr. Patrick Greiffenstein}

It would not appear so in the case description provided. In my limited search (PubMed, Cochrane libraries) no association between NSTI and risk of aortic aneurysmal disease has been described.

\section{Question 4: Whether the patient may have an underlying disease that worsens the infection and subsequent appearance of thoracic aortic aneurysm? Expert opinion 1: Dr. Francesco Petrella}

Aortic aneurysm can be more frequently observed in patients with underlying aortic wall disease like Marfan syndrome or collagenopathies; anyway the two events can be completely unrelated. Some more information could come from autopsy, if performed.

\section{Expert opinion 2: Dr. Giuseppe Marulli}

Diabetes, alcohol abuse and immunodeficiency are well known predictors of infection risks. In this particular case, however, I have some concerns about the first intervention of lobectomy and the subsequent redo-thoracotomy due to bleeding. It could be interesting to revise the description of surgery in order to understand if the lung was damaged or opened during dissection due to adhesions and a possible spread of pathogens infected the chest cavity. We have to remind that the patient was operated for bronchiectasis and probably the lung was infected not only by Aspergillus but also by other multi-drug resistant pathogens.

\section{Expert opinion 3: Dr. Fae fun Kim}

Generally, underlying diseases, such as chronic illnesses (chronic inflammatory disease and malignancy), malnutrition, hyperglycemia, and conditions associated with prolonged intake of corticosteroids and other infection at locations remote from the surgical, are known to worsen infection. Worsening infection is associated with subsequent appearance of thoracic aortic aneurysm.

\section{Expert opinion 4: Dr. Patrick Greiffenstein}

The only clear association between an aortic aneurysm and a chronic, insidious infectious process is that present in the 
tertiary stages of syphilitic disease (treponema pallidum) which causes endarteritis obliterans of the aortic wall leading to rupture if untreated. Primary infected aortic aneurysm is an exceedingly rare condition typically present in immunocompromised individuals. Clinical presentation includes the presence of signs of infection such as fever and leukocytosis and imaging suggestive of paraaortic inflammation (8). In the absence of this description or pathological analysis confirmatory of an infection of the region of the aorta in question, I would suggest that the two disease processes are causally unrelated.

\section{Conclusions}

Post-lobectomy infection involving chest wall may produce a unique diagnostic and therapeutic challenge, especially in the circumstances of unidentified pathogen. Complete surgical debridement of all grossly affected tissue is an effective and definitive treatment, with use of wide spectrum antibiotic therapy. Several newly-developed techniques, i.e. next generation sequencing, can help identify the causative microorganism.

\section{Acknowledgments}

We thank the nurses in the Department of Thoracic Surgery for their cooperation during the treatment of this patient.

Funding: This study was supported by the National Natural Science Foundation of China (Grant No. 81802260), Shanghai Rising-Star Program (Grant No. 19QA1407400), Shanghai Municipal Education Commission "Chen Guang" project (19CG19), Shanghai "Rising Stars of Medical Talent" Youth Development Program Youth Medical Talents-Specialist Program, Shanghai Pulmonary Hospital Fund for Excellent Young Scholars (No. fkyq1908).

\section{Footnote}

Reporting Checklist: The authors have completed the CARE reporting checklist. Available at http://dx. doi. org/10. 21037/jtd-20-2946

Conflicts of Interest: All authors have completed the ICMJE uniform disclosure form (available at http://dx. doi. org/10.21037/jtd-20-2946). PG reports personal fees from
Zimmer Biomet, outside the submitted work. JD serves as an unpaid Section Editor of Fournal of Thoracic Disease. The authors have no other conflicts of interest to declare.

Ethical Statement: The authors are accountable for all aspects of the work in ensuring that questions related to the accuracy or integrity of any part of the work are appropriately investigated and resolved. All procedures performed in studies involving human participants were in accordance with the ethical standards of the institutional and/or national research committee(s) and with the Helsinki Declaration (as revised in 2013). Written informed consent was obtained from the patient for publication of this manuscript and any accompanying images.

Open Access Statement: This is an Open Access article distributed in accordance with the Creative Commons Attribution-NonCommercial-NoDerivs 4.0 International License (CC BY-NC-ND 4.0), which permits the noncommercial replication and distribution of the article with the strict proviso that no changes or edits are made and the original work is properly cited (including links to both the formal publication through the relevant DOI and the license). See: https://creativecommons.org/licenses/by-nc-nd/4.0/.

\section{References}

1. Bagheri R, Haghi SZ, Fattahi MS, et al. Surgical management of bronchiectasis: analysis of 277 patients. Thorac Cardiovasc Surg 2010;58:291-4.

2. Imperatori A, Nardecchia E, Dominioni L, et al. Surgical site infections after lung resection: a prospective study of risk factors in 1,091 consecutive patients. J Thorac Dis 2017;9:3222-31.

3. Schipper P, Tieu BH. Acute Chest Wall Infections: Surgical Site Infections, Necrotizing Soft Tissue Infections, and Sternoclavicular Joint Infection. Thorac Surg Clin 2017;27:73-86.

4. Bergeron EJ, Meguid RA, Mitchell JD. Chronic Infections of the Chest Wall. Thorac Surg Clin 2017;27:87-97.

5. Barbosa RF, Pinho CJ, Costa-Ferreira A, et al. Microsurgical reconstruction of chest wall defect after necrotizing fasciitis. Microsurgery 2006;26:519-23.

6. Iarussi T, Marolla A, Pardolesi A, et al. Sternectomy and sternum reconstruction for infection after cardiac surgery. Ann Thorac Surg 2008;86:1680-1. 
7. Rudkjobing VB, Thomsen TR, Xu Y, et al. Comparing culture and molecular methods for the identification of microorganisms involved in necrotizing soft tissue infections. Bmc Infect Dis 2016;16:652.

Cite this article as: Dai J, Greiffenstein K, Petrella F, Kim JJ, Marulli G, Fang Y, Zhou Y. Treatment of a lung lobectomy patient with severe post-surgical infection in the anterior thoracic wall by multiple debridement and drainage procedures: a case report. J Thorac Dis 2020;12(12):7481-7487. doi: $10.21037 /$ jtd-20-2946
8. Hsu RB, Chen RJ, Wang SS, et al. Infected aortic aneurysms: clinical outcome and risk factor analysis. J Vasc Surg 2004;40:30-5. 